\title{
Dynamic logics of knowledge and access
}

\author{
Tomohiro Hoshi • Eric Pacuit
}

Received: 8 September 2009 / Accepted: 23 July 2010 / Published online: 27 August 2010

(C) The Author(s) 2010. This article is published with open access at Springerlink.com

\begin{abstract}
A recurring issue in any formal model representing agents' (changing) informational attitudes is how to account for the fact that the agents are limited in their access to the available inference steps, possible observations and available messages. This may be because the agents are not logically omniscient and so do not have unlimited reasoning ability. But it can also be because the agents are following a predefined protocol that explicitly limits statements available for observation and/or communication. Within the broad literature on epistemic logic, there are a variety of accounts that make precise a notion of an agent's "limited access" (for example, Awareness Logics, Justification Logics, and Inference Logics). This paper interprets the agents' access set of formulas as a constraint on the agents' information gathering process limiting which formulas can be observed.
\end{abstract}

Keywords Dynamic epistemic logic · Logics of awareness ·

Epistemic temporal logic

\section{Introduction and motivation}

Reasoning about rational agents interacting over time is a central topic in many areas of philosophy, computer science and economics. An important challenge for the logician

Eric Pacuit is supported by the NWO Vidi grant 016.094.345.

\section{T. Hoshi}

Department of Philosophy, Stanford University, Stanford, CA, USA

e-mail: thoshi@stanford.edu

E. Pacuit $(\bowtie)$

Tilburg Center for Logic and Philosophy of Science, Tilburg University, Tilburg, The Netherlands e-mail: e.j.pacuit@uvt.nl 
is to account for the many dynamic processes that govern the agents' interaction over time. Inference, observation and communication are all examples of such processes that are the focus of current logics of informational update and belief revision (see for example, van Benthem 1996; van Ditmarsch et al. 2007; Parikh and Ramanujam 2003). ${ }^{1}$ A recurring issue in any formal model representing agents' (changing) informational attitudes is how to account for the fact that the agents are limited in their access to the available inference steps, possible observations and available messages. This may be because the agents are not logically omniscient and so do not have unlimited reasoning ability. But it can also be because the agents are following a predefined protocol that explicitly limits statements available for observation and/or communication.

Within the broad literature on epistemic logic, there are a variety of accounts that make precise a notion of an agent's "limited access". An early approach of Fagin and Halpern $(1988)^{2}$ extends standard epistemic logic with an awareness operator $A \varphi$ intended to mean "the agent is aware of the sentence $\varphi$ ". More recently, work building on Artemov's Logic of Proofs (Artemov 2001) labels epistemic modal operators with proof terms that explicitly keep track of the agent's "justification" for (the truth of) a formula (see Fitting 2005; Artemov and Nogina 2005; Renne 2008, and references therein). Other logics focus on explicitly modeling inferential steps that individual agents can make while interacting with other agents and the environment (see for example, Eberle 1974; Ågotnes and Alechina 2007; van Benthem 2008; VelazquezQuesada 2009). Finally, van Benthem et al. (2009) develop logics for reasoning about situations where the facts that agents can observe are limited by a predefined protocol (cf. Parikh and Ramanujam 2003). Although the logical frameworks referenced above do differ in both "implementation details" and some underlying intuitions, there is a common thought that represents "what an agent currently has access to" as a set of formulas. We call this set of formulas the agent's access set.

There are two interpretations of this access set that can be found in the literature. The first views the access set as the (current) outcome of some information gathering process. That is, agents only have direct access to formulas that they have explicitly added to the access set (typically as a result of a logical inference step or observation, but other-trusted - agents may contribute to the access set through communication). This is the interpretation of the access sets found in the awareness logics (Halpern and Rego 2005), justification logics (Artemov and Nogina 2005; Fitting 2005), inference logics (Ågotnes and Alechina 2007; van Benthem 2008; Velazquez-Quesada 2009) and other epistemic logics with "explicit" knowledge operators. The second interpretation, found in van Benthem et al. (2009), uses the access set to constrain the current information gathering process. That is, the access set consists of the sentences that an agent $\mathrm{can}^{3}$ observe or infer.

\footnotetext{
1 Of course, one may argue that (logical) inference is the central topic of any logic. What we have in mind here is reasoning about agents that make inferences.

2 See also (Halpern and Rego 2005; Halpern 2001) for a recent discussion and references to relevant literature on the notion of awareness in game theory.

3 We may also say "is permitted to" or "has the ability to" (cf. Balbiani et al. forthcoming).
} 
Formally, the access set is a new parameter that is added to standard epistemic models. ${ }^{4}$ This suggests a number of technical and conceptual questions (especially in social situations involving many agents):

1. How should we extend the basic modal language to reason about epistemic models with access sets? Of course, the answer here depends on the intended interpretation of the access sets and the type of properties that need to be expressed. To give just two examples: Halpern and Rego (2009) use a propositional modal language with quantifiers over propositions and Artemov and others use a modal language with labeled modalities where each label refers to a different access set (cf. Artemov and Nogina 2005; Fitting 2005).

2. What do the agents "know" about the other agents' access sets? Again concrete answers to this question depend on the intended interpretation of the access sets. For example, if the access set represents the set of formulas an agent is "aware of", then certainly an agent cannot know that another agent is aware of a specific formula $\varphi$ without that agent being aware of $\varphi$. On the other hand, when access sets are "generated" by some underlying protocol or social convention it is natural to study situations where that underlying protocol may or may not be commonly known among the agents.

3. What dynamic operations change the access sets over time? A common assumption in the literature is that formulas are added to the access sets as a result of some logical inference (cf. Ågotnes and Alechina 2007). However, access sets may also change as a result of observation and/or communication. There is now an extensive literature on so-called dynamic epistemic logics describing different "epistemic actions" changing the agents' (implicit) information (both van Ditmarsch et al. (2007), and van Benthem (2010), are recent textbook presentations of this literature). Building on this literature, van Benthem (2008) studies logics with epistemic actions such as "becoming informed that..." or "seeing that..." which change the access sets (Velazquez-Quesada 2009; van Ditmarsch and French 2010; Hill 2010).

Much of the epistemic logic literature incorporating some version of an access set has focused on the first interpretation where access sets represent the agents' current stock of "available formulas". And so, each of the above questions have been addressed with this interpretation in mind. In this paper, we focus on the second interpretation where the access sets constrain the agents' social interactions and information gathering processes due to some underlying protocol or social convention. This continues a line of research initiated in (van Benthem et al. 2009, Sect. 4). There a constrained public announcement logic, where the formulas that can be observed (or announced) are restricted by some predefined protocol, is axiomatized.

We have two main goals in this paper. The first is to investigate the above questions (especially questions 1 and 2) in contexts where the agents' access sets are given by some underlying protocol. As mentioned above, this leads to interesting new technical results building on the work in van Benthem et al. (2009). Our main technical

\footnotetext{
4 Typically, a set of formulas is assigned to each agent at each state in an epistemic model. There is often additional structure placed on these sets (cf. Halpern 2001 and Fitting 2005). Such technical details are not important for the discussion in this section, though they will play a role in Sect. 3.5.
} 
contribution is an axiomatization where semi-private announcements are constrained by a predefined protocol (Sect. 3.3). Our second goal is to formally relate the logical framework that we investigate in this paper with similar frameworks found in the literature. In some cases, this leads to technical results showing our models are specific cases of more general "temporal dynamic epistemic models" as discussed in van Benthem et al. (2009); Hoshi (2009). We discuss this in Sect. 3.2. Comparisons with frameworks where the access sets are interpreted as outcomes of some information gathering processes (e.g., Awareness Logics and Justifications Logics) are not as direct. Nonetheless, Sect. 4 points to a number of conceptual issues suggested by our logical framework.

\section{Background}

Many logical systems today describe some form of "information dynamics". However, two main approaches can be singled out. The first is exemplified by epistemic temporal logic (ETL, Fagin et al. 1995; Parikh and Ramanujam 2003) which uses linear or branching time models with added epistemic structure induced by the agents' different capabilities for observing events. These models provide a "grand stage" where histories (i.e., sequences of events) of some social situation are constrained by a protocol. Here a protocol is intended to represent the rules or conventions that govern many of our social interactions. Imposing such rules restricts the legitimate sequences of possible events (e.g., messages or observations). The other approach is exemplified by dynamic epistemic logic (DEL, Gerbrandy 1999; Baltag et al. 1998; van Ditmarsch et al. 2007) which describes social interactions in terms of epistemic event models (which may occur inside modalities of the language). Similar to the way epistemic models are used to capture the (hard) information the agents' have about a fixed social situation, an event model describes the agents' information about which actual event is currently taking place. The temporal evolution of the situation is then computed from some initial epistemic model through a process of successive "product updates". Consult van Benthem et al. (2009); Kooi and Pacuit (2010) for an extensive discussion of these two perspectives on rational interaction.

We assume the reader is familiar with standard epistemic logic and the various dynamic versions mentioned above. Indeed, there are now a number of textbooks and survey papers that have thorough introductions to these topics (see for example, Fagin et al. 1995; van Ditmarsch et al. 2007; van Benthem 2010; Pacuit forthcoming; and references therein). In this Section, we discuss some key definitions used in this paper. We start with the familiar definition of an epistemic model:

Definition 1 Let At be a set of atomic propositions. An epistemic model is a tuple $\left\langle W,\left\{R_{i}\right\}_{i \in \mathcal{A}}, V\right\rangle$ where $W$ is a nonempty set (whose elements are called worlds or states), for each $i \in \mathcal{A}, R_{i} \subseteq W \times W$ is a relation ${ }^{5}$ and $V$ is a valuation function $\left(V:\right.$ At $\left.\rightarrow 2^{W}\right)$.

\footnotetext{
5 Typically, it is assumed that these relations are equivalence relations, but this is not necessary.
} 
An ETL model is a special case of an epistemic model where the states are sequences of primitive events. That is, given a finite nonempty set $\Sigma$ (whose elements are called events), an ETL model (based on $\Sigma$ ) is a tuple $\left\langle\mathrm{H},\left\{R_{i}\right\}_{i \in \mathcal{A}}, V\right\rangle$ where $\left\{R_{i}\right\}_{i \in \mathcal{A}}$ and $V$ are as in the above definition and $\mathrm{H} \subseteq \Sigma^{*}$ is closed under non-empty prefixes. Elements of $\mathrm{H}$ are called histories and are intended to represent a possible way that a social situation may unfold. So, ETL models are forests (in general, there need not be a unique initial state) with relations for each agent on the set of histories (for this reason we often refer to ETL models as ETL forests). Different modal languages describe ETL models (see for example, Hodkinson and Reynolds 2006; Fagin et al. 1995), with 'branching' or 'linear' variants. As an example, the language $\mathcal{L}_{E T L}$ contains both knowledge and "event" modalities:

$$
p|\neg \varphi| \varphi \wedge \psi\left|K_{i} \varphi\right|\langle e\rangle \varphi
$$

where $i \in \mathcal{A}, e \in \Sigma$ and $p \in$ At (the set of atomic propositions). The boolean connectives $(\vee, \rightarrow, \leftrightarrow)$ and the dual modal operators $\left(L_{i},[e]\right)$ are defined as usual. The intended interpretation of ' $\langle e\rangle \varphi$ ' is "after event $e$ (does) take place, $\varphi$ is true". Formulas are interpreted at histories: let $\mathcal{H}=\left\langle\mathrm{H},\left\{R_{i}\right\}_{i \in \mathcal{A}}, V\right\rangle$ be an ETL model, $\varphi \in \mathcal{L}_{E T L}$ and $h \in \mathrm{H}$, define $\mathcal{H}, h \models \varphi$ inductively as follows (we only give the modal definitions as the boolean connectives and atomic propositions are as usual),

1. $\mathcal{H}, h \models K_{i} \varphi$ iff for each $h^{\prime} \in \mathrm{H}$, if $h R_{i} h^{\prime}$ then $\mathcal{H}, h^{\prime} \models \varphi$

2. $\mathcal{H}, h \models\langle e\rangle \varphi$ iff $h e \in \mathrm{H}$ and $\mathcal{H}$, he $\models \varphi$

The epistemic language, denoted $\mathcal{L}_{E L}$, is the sublanguage of $\mathcal{L}_{E T L}$ without the event modalities. Natural extensions of $\mathcal{L}_{E T L}$ include group operators (e.g., common or distributed knowledge) and more expressive temporal operators (e.g., arbitrary future or past modalities).

A key observation from van Benthem et al. (2009) is that we can generate ETL models from an initial epistemic model using the machinery of DEL. This opens the door to rigorously comparing and merging the two main logical accounts of the dynamics of information in social interactive situations. Formally, a DEL protocol is a tree of event models (representing the complex informative events that are available at each moment). Given an epistemic model $\mathcal{M}=\left\langle W,\left\{R_{i}\right\}_{i \in \mathcal{A}}, V\right\rangle$ and a DEL protocol $\mathrm{P}$, Forest $(\mathcal{M}, \mathrm{P})$ is the ETL model representing all possible evolutions of the system obtained by updating $\mathcal{M}$ with sequences from $\mathrm{P}$. We do not include the details of this general construction here (see van Benthem et al. 2009, Definitions 8 and 10). Instead we briefly discuss constrained public announcement logic which reexamines public announcement logic (PAL, Gerbrandy 1999; Plaza 1989) in situations where the availability of formulas for observation is constrained by a predefined protocol.

A public announcement is an event where some (epistemic) formula $\varphi$ is made publicly available. That is, it is completely open and all agents not only observe the event but also observe everyone else observing the event, and so on ad infinitum. Furthermore, all agents treat the source as infallible. Thus the effect of such an event on an epistemic model should be clear: remove all states that do not satisfy $\varphi$. Formally,

Definition 2 Suppose $\mathcal{M}=\left\langle W,\left\{R_{i}\right\}_{i \in \mathcal{A}}, V\right\rangle$ is an epistemic model and $\varphi$ is a (epistemic) formula. The model updated by the public announcement of $\varphi$ is the 
structure $\mathcal{M}^{\varphi}=\left\langle W^{\varphi},\left\{R_{i}^{\varphi}\right\}_{i \in A}, V^{\varphi}\right\rangle$ where $W^{\varphi}=\{w \in W \mid \mathcal{M}, w \models \varphi\}$, for each $i \in \mathcal{A}, R_{i}^{\varphi}=R_{i} \cap W^{\varphi} \times W^{\varphi}$, and for all atomic proposition $p, V^{\varphi}(p)=V(p) \cap W^{\varphi}$.

The language of PAL extends $\mathcal{L}_{E L}$ with dynamic modal operators, $\langle\varphi\rangle \psi$, meaning "after $\varphi$ is publicly announced, $\psi$ is true". These modal operators are interpreted on epistemic models as follows:

$$
\mathcal{M}, w \models\langle\varphi\rangle \psi \quad \text { iff } M, w \models \varphi \quad \text { and } \quad \mathcal{M}^{\varphi}, w \models \psi \text {. }
$$

A PAL protocol is a tree of (epistemic) formulas. Given an epistemic model $\mathcal{M}$ and PAL protocol $\mathrm{P}$, an ETL model Forest $(\mathcal{M}, \mathrm{P})$ can be generated by performing public announcements of formulas permitted by P. ${ }^{6}$ More concretely, a sequence of epistemic formulas $\sigma=\varphi_{1} \cdots \varphi_{n}$ with an initial epistemic model generates a sequence of epistemic models

$$
\mathcal{M}, \mathcal{M}^{\varphi_{1}},\left(\mathcal{M}^{\varphi_{1}}\right)^{\varphi_{2}}, \ldots,\left(\left(\left(\mathcal{M}^{\varphi_{1}}\right)^{\varphi_{2}}\right)^{\cdots}\right)^{\varphi_{n}}
$$

This can be turned into an ETL model with histories of the form $w \varphi_{1} \varphi_{2} \cdots \varphi_{j}$ where $w$ is a state in $\mathcal{M}$ and $\mathcal{M}, w \models \bigwedge_{1 \leq i \leq j} \varphi_{i}$. Then Forest $(\mathcal{M}, \mathrm{P})$ consists of all such histories consistent with $P$.

In this new setting the PAL formula $\langle\varphi\rangle \top$ not only expresses that the current model $i s$ updated by the public announcement of $\varphi$ but also that $\varphi$ is permitted according to the predefined PAL protocol. Taking into account this new interpretation of the PAL language, van Benthem et al. (2009) give a sound and complete axiomatization of the class of all ETL models generated by some epistemic model and PAL protocol. ${ }^{7}$

A public announcement is one specific type of event model, what about classes of ETL models generated by other types of event models? That is, given a set of DEL protocols $\mathbf{X}$, can we axiomatize the class $\mathbb{F}(\mathbf{X})=\{\operatorname{Forest}(\mathcal{M}, \mathrm{P})$ $\mathcal{M}$ an epistemic model and $\mathrm{P} \in \mathbf{X}\}$ ? Three parameters are crucial for such a logical analysis:

1. The type of epistemic events in the protocol(s). Examples range from public announcements (Definition 2) where everyone witnesses the same event to private communications between a group of agents with the other agents not even being aware of the event.

2. Structural properties of the protocol(s). Examples range from restricting trees to a fixed length to "fairness properties" such as if the agent can observe $\varphi$, then the agent can observe $\neg \varphi$.

3. Expressivity of the formal language used to describe these DEL generated ETL models.

\footnotetext{
${ }^{6}$ Actually the most general situation is where there are different PAL protocols at each state. We return to this issue in Sect. 3.3.

${ }^{7}$ Note that the usual method for proving completeness via reduction axioms will not work here. In particular, since $\varphi$ being true does not necessarily imply that $\varphi$ can be announced and so the PAL validity $\varphi \leftrightarrow\langle\varphi\rangle \top$ is not valid (of course, $\langle\varphi\rangle \top \rightarrow \varphi$ is valid since we are working with true announcements).
} 
There is a growing literature providing logical analyses along these lines although the general situation is not yet fully understood. It is beyond the scope of this paper to provide a complete survey of this literature: see van Benthem et al. (2009) for a discussion. This paper contributes to this literature in two ways. First, we axiomatize $\mathbb{F}(\mathbf{X})$ where $\mathbf{X}$ is the class of semi-private announcements satisfying various structural properties. Second, we suggest that the formal language used to describe ETL models of the from Forest $(\mathcal{M}, \mathrm{P})$ should contain three different types of operators: (1) operators describing the agents' informational attitudes (knowledge, beliefs, group notions), (2) temporal and/or dynamic operators and (3) operators that can describe the protocol.

\section{Protocols of semi-private announcements}

We assume that at each moment different pieces of information are made publicly available. However, as opposed to public announcements where all agents have access to this information, we assume that different agents have access to different pieces of information. Thus we are interested in ETL models generated by protocols consisting of the following type of event model:

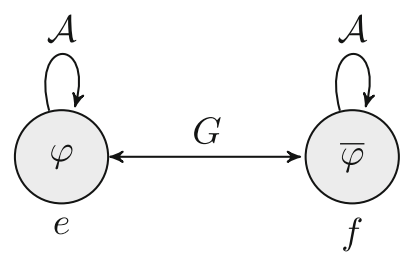

where $\mathcal{A}$ is the (finite) set of agents, $\varphi$ is a formula (in the language defined below) and $\bar{\varphi}$ is the "negation" of $\varphi$ (i.e., $\bar{\varphi}=\psi$ if $\varphi=\neg \psi$ and $\bar{\varphi}=\neg \varphi$ otherwise). This event model represents situations where (the truth of) $\varphi$ is made available only to the agents $\mathcal{A}-G$. Of course, the event itself is public so which agents actually have access to which pieces of information is commonly known (i.e., the agents in $G$ know that the agents in $\mathcal{A}-G$ know whether $\varphi$ is true).

The logical framework discussed in the previous section based on van Benthem et al. (2009) is very general focusing on ETL models generated by arbitrary event models. Note that when working with a specific type of event model (e.g., public announcement or semi-private announcement), many of the definitions can be simplified (cf. the constrained public announcement logic from Sect. 4 of van Benthem et al. 2009). These simplified versions also facilitate a more direct comparison with various awareness logics discussed in Sect. 1. The precise relationship with the more general framework of van Benthem et al. (2009) is given in Sect. 3.2.

\subsection{A dynamic logic of knowledge and access}

The Language. The language of the Dynamic Logic of Knowledge and Access (DKA) includes standard epistemic and dynamic modalities plus operators intended to describe each agent's access set, or protocol (i.e., which formulas the agent has 
access to). Fix a finite set $\mathcal{A}$ of agents and a (countable) set of atomic propositions At. The language $\mathcal{L}_{D K A}$ is defined inductively:

$$
\varphi::=\top|p| \neg \varphi|\varphi \wedge \varphi| K_{i} \varphi|\langle\varphi\rangle \varphi| A_{i} \varphi
$$

where $p \in$ At and $i \in \mathcal{A}$. The dual operators, $L_{i}$ and $[\varphi]$, and other boolean operators are defined as usual. The epistemic fragment of $\mathcal{L}_{D K A}$ without the ' $\langle\varphi\rangle$ ' and ' $A_{i}$ ' operators is denoted $\mathcal{L}_{E L}$. The intended meaning of the modal operators is summarized below:

- $K_{i} \varphi$ is intended to mean "according to $i$ 's current information $\varphi$ is true" (following the standard convention we may also say "agent $i$ knows that $\varphi$ ").

- $\langle\varphi\rangle \psi$ is intended to mean "after $\varphi$ is made publicly available, $\psi$ is true" (we may also say "after $\varphi$ is announced $\psi$ is true", but this should not be confused with the public announcement of $\varphi$ discussed in the previous section.

- $A_{i} \varphi$ is "agent $i$ has access to $\varphi$ " (alternatively we may say "agent $i$ can observe $\varphi$ " or "agent $i$ has the ability to observe $\varphi$ ").

For later use, we define the complexity $c(\varphi)$ of a formula $\varphi$ :

Definition 3 The complexity of $\varphi$, denoted $c(\varphi)$ is defined as follows: $c(p)=0$ where $p \in$ At, $c(\alpha \wedge \beta)=c(\alpha)+c(\beta)+1, c(\neg \alpha)=1+c(\alpha), c\left(K_{i} \alpha\right)=1+c(\alpha), c\left(A_{i} \alpha\right)=$ $1+c(\alpha)$ and $c(\langle\alpha\rangle \beta)=c(\alpha)+c(\beta)+1$.

The Semantics. Our models extend epistemic models with a description of what the agents can observe. Note that "being able to observe $\varphi$ " is an event type which we take to mean that in situations where $\varphi$ is true, the agent observes that $\varphi$ and when $\varphi$ is false the agent observes that $\neg \varphi$. This explains the closure condition in the following definition:

Definition 4 A protocol is a function $\mathrm{p}: \mathcal{A} \times \mathbb{N} \rightarrow \wp\left(\mathcal{L}_{D K A}\right)$ such that, for every $n \in \mathbb{N}, i \in \mathcal{A}$, and $\varphi \in \mathcal{L}_{D K A}, \varphi \in \mathrm{p}(i, n)$ iff $\bar{\varphi} \in \mathrm{p}(i, n)$. We denote the set of protocols by Ptcl. For brevity, we write $\mathrm{p}_{i}^{n}$ for $\mathrm{p}(i, n)$ where $\mathrm{p} \in P t c l, i \in \mathcal{A}$, and $n \in \mathbb{N}$.

Using the terminology from Sect. 1, a protocol describes for each agent and each moment which formulas that agent can access. So, in this setting, the agents' access sets consist of sequences of formulas representing which observations are available now and in the future (and in what order). The closure condition states that agents have access to a formula iff they have access to its "negation". Of course, this is only one of many different properties that may be assumed about the protocol. For example, it may be natural to assume that if an agent has access to both $\varphi$ and $\psi$ then the agent must also have access to $\varphi \wedge \psi$. Using the access modality $A_{i}$ such properties will be expressible in our language. This will be discussed in more detail in Sect. 3.5.

Definition 5 An epistemic model with a protocol is a quadruple $\left\langle W,\left\{R_{i}\right\}_{i \in \mathcal{A}}, V, \mathrm{p}\right\rangle$, where $\left\langle W,\left\{R_{i}\right\}_{i \in \mathcal{A}}, V\right\rangle$ is an epistemic model (Definition 1) and $\mathrm{p}$ is a protocol (Definition 4). 
We are restricting attention to one type of dynamic epistemic action: "making $\varphi$ publicly available where only some of the agents have access to (the observation of) $\varphi$ ". Here "having access to $\varphi$ " means that the agent can incorporate the observation of $\varphi$ into the agent's current information. Formally, "making $\varphi$ publicly available" amounts to performing a product update (Baltag et al. 1998) with the semi-private announcement of $\varphi$ using the event model given above (where $G$ is the set of agents who do not have $\varphi$ in their protocol). In this simplified setting, we can define this update as a restriction on the agents' current accessibility relations. Intuitively, if an agent incorporates the observation of $\varphi$, she should consider possible only states where $\varphi$ is true. But in order to do this, the agent must have access to both $\varphi$ and $\bar{\varphi}$ (this also explains the closure condition placed on protocols in Definition 4). So, the accessibility relation does not change for agents without access to $\varphi$. For agents with access to $\varphi$, all connections between $\varphi$ and $\neg \varphi$ worlds are dropped.

Definition 6 Let $\mathcal{P}=\left\langle W,\left\{R_{i}\right\}_{i \in \mathcal{A}}, V, \mathrm{p}\right\rangle$ be an epistemic model with a protocol. The truth of a formula $\varphi$ in $\mathcal{L}_{D K A}$ is defined as follows:

$$
\begin{aligned}
& \mathcal{P}, w \models p \quad \text { iff } w \in V(p) \quad \text { (with } p \in P \text { ) } \\
& \mathcal{P}, w \models \neg \varphi \quad \text { iff } \mathcal{P}, w \not \models \varphi \\
& \mathcal{P}, w \models \varphi \wedge \psi \text { iff } \mathcal{P}, w \models \varphi \text { and } \mathcal{P}, w \models \psi \\
& \mathcal{P}, w \models K_{i} \varphi \quad \text { iff } \forall v \in W: \text { if } w R_{i} v \text { then } \mathcal{P}, v \models \varphi \\
& \mathcal{P}, w \models\langle\varphi\rangle \psi \quad \text { iff } \mathcal{P}, w \models \varphi \text { and } \mathcal{P} \otimes \varphi, w \models \psi \\
& \mathcal{P}, w \models A_{i} \varphi \quad \text { iff } \varphi \in \mathrm{p}(i, 0)
\end{aligned}
$$

where $\mathcal{P} \otimes \varphi=\left\langle W^{\prime},\left\{R^{\prime}\right\}_{i \in \mathcal{A}}, V^{\prime}, \mathrm{p}^{\prime}\right\rangle$ is defined by:

$$
\begin{aligned}
& W^{\prime}:=W \\
& R_{i}^{\prime}:= \begin{cases}R_{i} & \text { if } \bar{\varphi} \notin \mathrm{p}(i, 0) \\
\left\{(w, v) \in R_{i} \mid \mathcal{P}, w \models \varphi \text { iff } \mathcal{P}, v \models \varphi\right\} & \text { if } \bar{\varphi} \in \mathrm{p}(i, 0)\end{cases} \\
& V^{\prime}(p):=V(p) \\
& \mathrm{p}^{\prime}(i, n):=\mathrm{p}(i, n+1)
\end{aligned}
$$

\subsection{Comparison with other systems}

We stress that there is nothing new in the definition of the above epistemic action. Indeed, it is a simple exercise to check that for any epistemic model $\mathcal{M}$ and formula $\varphi, \mathcal{M} \otimes \varphi$ is isomorphic to the model resulting from the product update of $\mathcal{M}$ and the event model:

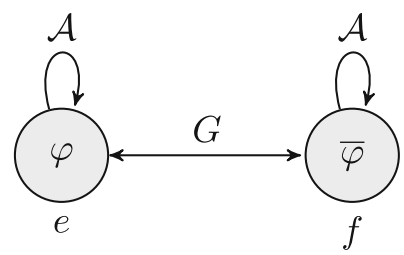


where $G=\left\{i \mid \varphi \notin \mathrm{p}_{i}^{0}\right\}$. In this section, we are more precise about the relationship between our framework and the logical systems discussed in Sect. 2.

It is easy to see that our framework generalizes public announcement logic (PAL). Let $\mathcal{L}_{P A L}$ be the set of PAL-formulas. Also let $\mathcal{P}_{P A L}=\left\langle W,\left\{R_{i}\right\}_{i \in \mathcal{A}}, V, \mathrm{p}\right\rangle$ be a model where for each $i \in \mathcal{A}$ and $n \in \mathbb{N}, \mathrm{p}(i, n)=\mathcal{L}_{P A L}$. It is a simple exercise to check that for each $\varphi \in \mathcal{L}_{P A L}$ :

$$
\mathcal{P}_{P A L}, w \models \varphi \text { in DKA } \quad \text { iff } \quad\left\langle W,\left\{R_{i}\right\}_{i \in \mathcal{A}}, V\right\rangle, w \models \varphi \text { in PAL. }
$$

So, in a PAL model, agents always have access to all formulas. Given this it should not be surprising that there are PAL validities that are falsifiable over the class of epistemic models with protocols:

Observation 1 The following are falsifiable:

1. $\langle\alpha\rangle\langle\beta\rangle \varphi \rightarrow\langle\langle\alpha\rangle \beta\rangle \varphi$

2. $[p] K_{i} p$ where $p \in$ At

3. $\langle\theta\rangle K_{i} \varphi \leftrightarrow\langle\theta\rangle \top \wedge K_{i}(\theta \rightarrow\langle\theta\rangle \varphi)$

Proof The first two formulas illustrate the role that the protocols play in our framework.

1. Formula 1 can be falsified by putting $\alpha=\beta:=p$ and $\varphi:=K_{i} p$. Let $\mathcal{P}=$ $\left\langle W,\left\{R_{i}\right\}_{i \in \mathcal{A}}, V, \mathrm{p}\right\rangle$ be a model where $\mathcal{P}, w \models p \wedge \neg K_{i} p$ for some $w$. If $p \in \mathrm{p}(i, 1)$ and $p \in \mathrm{p}(i, 2)$, but $\langle p\rangle p \notin \mathrm{p}(i, 1)$, we have $\mathcal{P}, w \models\langle p\rangle\langle p\rangle K_{i} p \wedge \neg\langle\langle p\rangle p\rangle K_{i} p$.

2. Formula 2 can be falsified at any state $w$ in $\mathcal{P}=\left\langle W,\left\{R_{i}\right\}_{i \in \mathcal{A}}, V, \mathrm{p}\right\rangle$ where $\mathcal{P}, w \models p \wedge \neg K_{i} p$ and $p \notin \mathrm{p}(i, 0)$.

3. Formula 3 is a critical formula for the reduction analysis in PAL, which can be falsified by putting $\theta=\varphi:=p$. Consider a model $\mathcal{P}=\left\langle W,\left\{R_{i}\right\}_{i \in \mathcal{A}}, V, \mathrm{p}\right\rangle$ and state $w$ where $\mathcal{P}, w \models p \wedge \neg K_{i} p$ but $p \notin \mathrm{p}(i, 0)$. Then the left-hand-side of the bi-conditional is false while the right-hand-side is true.

However, there is an embedding from the language of PAL to $\mathcal{L}_{D K A}$ that preserves validity: let $\operatorname{sub}(\varphi)$ be the set of subformulas of $\varphi$,

Proposition 1 For every formula $\varphi$ of PAL,

$$
\varphi \text { is valid in PAL iff }\left(\bigwedge_{i \in \mathcal{A}} \bigwedge_{\psi \in \operatorname{sub}(\varphi)} A_{i} \psi\right) \rightarrow \varphi \text { is valid in } \mathrm{DKA}
$$

The simple but instructive proof is left to the reader (but the intuition is clear: the dynamic operation "make $\varphi$ public" from Definition 6 behaves like a public announcement of $\varphi$ provided all agents have access to all subformulas of $\varphi$ ).

We conclude this section with a precise comparison with temporal dynamic epistemic logic as discussed in Sect. 2 (cf. also Hoshi 2009; Hoshi and Yap 2009). In DKA, when $\varphi$ is made publicly available, the agents may or may not obtain the information 
that $\varphi$, depending on whether they have access to $\varphi$. As discussed above, this situation can be modeled by an event model that consists of two events, whose preconditions are $\varphi$ and $\neg \varphi$, respectively. Only agents who have access to $\varphi$ (and $\neg \varphi$ ) can distinguish these two events. We give a formal translation below.

Let $\mathrm{p}$ be a protocol and $\varphi$ a formula in $\mathcal{L}_{D K A}$. Define an event $\operatorname{model}^{8} \mathcal{E}(\varphi, \mathrm{p}, n)=$ $\left\langle E,\left\{\rightarrow_{i}\right\}_{i \in \mathcal{A}}\right.$, pre $\rangle$ as follows:
1. $E=\{1,2\}$
2. $\rightarrow_{i}= \begin{cases}\{(1,1),(1,2),(2,1),(2,2)\} & \text { if } \varphi \in \mathrm{p}(i, n) \\ \{(1,1),(2,2)\} & \text { if } \varphi \notin \mathrm{p}(i, n) .\end{cases}$
3. $\operatorname{pre}(1)=\varphi, \operatorname{pre}(2)=\neg \varphi$

Given a protocol model $\mathcal{P}=\left\langle W,\left\{R_{i}\right\}_{i \in \mathcal{A}}, V, \mathrm{p}\right\rangle$, we define a DEL generated ETL model $\operatorname{tr}(\mathcal{P})=\operatorname{Forest}\left(\mathcal{M}^{\mathcal{P}}, \mathrm{p}^{\mathcal{P}}\right)$ as follows:

1. $\mathcal{M}^{\mathcal{P}}=\left\langle W,\left\{R_{i}\right\}_{i \in \mathcal{A}}, V\right\rangle$ and

2. $\mathrm{p}^{\mathcal{P}}$ is a state-dependent protocol ${ }^{9}$ on $\mathcal{M}^{\mathcal{P}}$ such that, for every $w \in W, \mathrm{p}^{\mathcal{P}}(w)$ consists of all sequences of the form $\sigma=\sigma_{0} \cdots \sigma_{n}$ where $\sigma_{k}(0 \leq k \leq n)$ is of the form $\langle\mathcal{E}(\varphi, \mathrm{p}, k), e\rangle$ with $e$ in $\mathcal{E}(\varphi, \mathrm{p}, k)$.

Let $\mathcal{L}_{D K A}^{-}$be the fragment of $\mathcal{L}_{D K A}$ without the operator $A_{i}$. We are now ready to give a formal translation from our language to the language of temporal dynamic epistemic logic. For each $k \in \mathbb{N}$ define $t r_{k}$ as follows: $t r_{k}(p)=p, t r_{k}$ commutes with the boolean connectives and the $K_{i}$ operators and

$$
\operatorname{tr}_{k}(\langle\varphi\rangle \psi)=\left\langle\mathcal{E}\left(\operatorname{tr}_{k}(\varphi), \mathrm{p}, k\right), 1\right\rangle \operatorname{tr}_{k+1}(\psi)
$$

Unpacking the above definitions gives us the following result: let $\models_{E T L}$ denote the truth relation for ETL models (see Sect. 2),

Proposition 2 For any epistemic model with a protocol and formula $\varphi \in \mathcal{L}_{D K A}$,

$$
\mathcal{P}, w \models \varphi \quad \text { iff } \quad \operatorname{tr}(\mathcal{P}), w \models E T L \operatorname{tr}_{0}(\varphi)
$$

\subsection{Axiomatization}

There are two main categories of axiom schemes. The first contains reduction axioms describing the effect of "making a formula publicly available" on an epistemic model:

R1 $\langle\theta\rangle p \leftrightarrow \theta \wedge p$ where $p \in$ At

R2 $\langle\theta\rangle \neg \varphi \leftrightarrow \theta \wedge \neg\langle\theta\rangle \varphi$

R3 $\langle\theta\rangle(\varphi \wedge \psi) \leftrightarrow\langle\theta\rangle \varphi \wedge\langle\theta\rangle \psi$

\footnotetext{
${ }^{8}$ An event model is a tuple $\left\langle E,\left\{\rightarrow_{i}\right\}_{i \in \mathcal{A}}\right.$, pre $\rangle$ where $E$ is a set of primitive events, for each $i \in \mathcal{A}, \rightarrow_{i}$ is a relation on $E$ and pre : $E \rightarrow \mathcal{L}_{E L}$. We assume the reader is familiar with this notion, the definition of product update and the language of DEL (Baltag et al. 1998; van Ditmarsch et al. 2007; van Benthem 2010).

${ }^{9}$ A state-dependent protocol on an epistemic model $\mathcal{M}$ assigns a possible different protocol to each state in the model.
} 


\section{R4 $\langle\theta\rangle K_{i} \varphi \leftrightarrow \theta \wedge\left(A_{i} \theta \rightarrow K_{i}[\theta] \varphi\right) \wedge\left(\neg A_{i} \theta \rightarrow K_{i}([\theta] \varphi \wedge[\bar{\theta}] \varphi)\right)$}

Two key observations about these axiom schemes are in order. First of all, note that $\langle\theta\rangle \top \leftrightarrow \theta$ is a consequence of $\mathrm{R} 1$. This means that any true formula can always be made publicly available. Of course, whether agents have access to this formula depends on their protocols. This distinguishes our framework from the one found in Sect. 4 of van Benthem et al. (2009) where $\langle\theta\rangle \top$ means both that $\theta$ is publicly announced and $\theta$ can be observed according to the protocol. Here $\langle\theta\rangle \top$ simply means that $\theta$ is made publicly available. We use the $A_{i}$ operator to express when agents have access to specific formula (i.e., $A_{i} \varphi$ means that $\varphi$ is in agent $i$ 's current protocol).

Second, strictly speaking, R4 may not be properly called a reduction axiom since the right-hand side of the biconditional increases the complexity (according to Definition 3) of the formula inside the announcement modality. Nonetheless, following the usual reduction axiom methodology, the right-hand side does describe what agents know after $\theta$ is made publicly available in terms of the agent's current information. When $\theta$ is made publicly available, there are two array to consider. The first is when agent $i$ does have access to $\theta$, so $A_{i} \theta$ is true. This case is covered by the second conjunct. When an agent has access to $\theta$, the links between $\theta$-worlds and $\neg \theta$-worlds are removed after the announcement of $\theta$. Thus, $\langle\theta\rangle K_{i} \varphi$ is the same as $K_{i}[\theta] \varphi$ following typical reduction axiom reasoning. The third conjunct describes the situation in which the agent does not have access to $\theta$ ( $\operatorname{so} \neg A_{i} \theta$ is true). In this case, the agent's accessibility relation will not change, so any links between $\theta$-worlds and $\neg \theta$-worlds will not be removed. Thus, agents will know the formulas that survive both an announcement of $\theta$ and an announcement of $\bar{\theta}$. This situation is described by the formula $K_{i}([\theta] \varphi \wedge[\bar{\theta}] \varphi)$.

To introduce the second category of axiom schemes, we need some notation. Let $\sigma$ be a (possibly empty) finite sequence of $\mathcal{L}_{D K A}$-formulas (we write this as $\sigma \in \mathcal{L}_{D K A}^{*}$ ). We denote the length of $\sigma$ by len $(\sigma)$. Also, we denote by $\sigma_{n}$ and $\sigma_{(n)}$ the $n$-th element of $\sigma$ and the initial segment of $\sigma$ of the length $n$, respectively. When $n$ is greater than the length of $\sigma$, the former denotes the empty sequence $\lambda$. Finally, we write $\bar{\sigma}$, $\langle\sigma\rangle$ and $[\sigma]$ for $\overline{\sigma_{1}} \ldots \overline{\sigma_{\operatorname{len}(\sigma)}},\left\langle\sigma_{1}\right\rangle \ldots\left\langle\sigma_{\operatorname{len}(\sigma)}\right\rangle$ and $\left[\sigma_{1}\right] \ldots\left[\sigma_{\operatorname{len}(\sigma)}\right]$, respectively. The following axiom schemes describe our assumptions about the protocols:

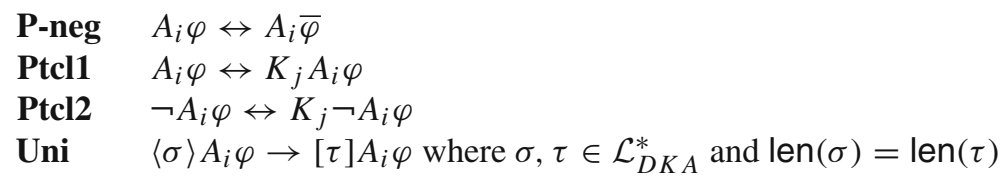

The first axiom scheme encodes the closure condition on protocols that agents have access to $\varphi$ iff they have access to $\bar{\varphi}$. The last three axiom schemes encode the fact that protocols are "common knowledge"10 and uniform. Ptcl1 \& Ptcl2 guarantee that every agent knows the access sets, and by standard modal reasoning, all agents know all agents know it,..., up to an arbitrary depth. ${ }^{11}$ Uni adds the additional constraint that the formulas an agent has access to do not depend on earlier observations.

\footnotetext{
10 Of course, since we do not have a common knowledge operator in our language it is only an informal statement that the protocol is common knowledge.

11 This is also guaranteed for the future access sets as well as the current access set, as will be shown in the proof of Lemma 2.
} 
That is, each agent's access set is determined only by the temporal point and not the history of previous observations. This assumption is built into our definition of a protocol as a function assigning sets of formulas to agent-moment pairs. ${ }^{12}$

\subsection{Completeness}

Our main technical contribution is a sound and complete axiomatization of the class of all epistemic models with protocols (cf. Definition 5) in the language $\mathcal{L}_{D K A}$. We first gather the axioms from the previous section in one definition:

Definition 7 (Axiomatization) The logic DKA is the smallest set containing all instances of the following axiom schemes: ${ }^{13}$

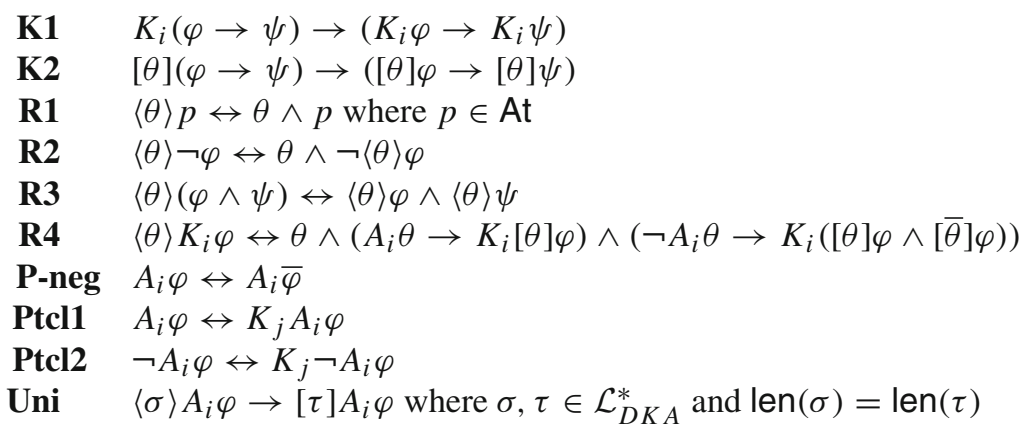

and is closed under necessitation for $K_{i}$ and $[\theta]$. We write $\vdash_{\operatorname{DKA}} \varphi$ if $\varphi \in \mathrm{DKA}$.

Our goal in this section is to prove the following result:

Theorem 1 DKA is sound and strongly complete with respect to the class of epistemic models with protocols.

Soundness is a simple (and instructive) exercise. The proof of completeness is a variant of the one found in Section 4 of van Benthem et al. (2009) which itself is a variant of the standard Henkin construction (cf. Section 4.2, Blackburn et al. 2002). We construct a canonical model from the set of DKA maximal consistent sets (MCS). A key observation is that each MCS contains a description of a protocol:

Definition 8 Given a maximally consistent set $\Gamma$, we define the $\Gamma$-protocol $\mathrm{p}^{\Gamma}$ as follows:

$$
\mathrm{p}^{\Gamma}(i, n)= \begin{cases}\left\{\varphi \mid A_{i} \varphi \in \Gamma\right\} & \text { if } n=0 \\ \left\{\varphi \mid \exists \psi_{1} \ldots \psi_{n}:\left\langle\psi_{1}\right\rangle \ldots\left\langle\psi_{n}\right\rangle A_{i} \varphi \in \Gamma\right\} & \text { if } n \geq 1\end{cases}
$$

\footnotetext{
12 We also note that in van Benthem et al. (2009) the existential modality is needed to express this uniformity property. We do not need it here given our definition of a model (Definition 5) and the more expressive language.

${ }^{13}$ For concreteness, we only include the axiom schema $K_{i}(\varphi \rightarrow \psi) \rightarrow\left(K_{i} \varphi \rightarrow K_{i} \psi\right)$, but other modal logics will work as well such as S5.
} 
That $\mathrm{p}^{\Gamma}$ is in fact a protocol according to Definition 4 is an immediate consequence of axiom P-neg and standard modal reasoning. Next we define the base canonical model that will serve as the initial epistemic model.

Definition 9 Let $\Gamma$ be a maximally consistent set. The $\Gamma$-generated canonical model $\mathcal{P}^{\Gamma}=\left\langle W^{\Gamma},\left\{R_{i}^{\Gamma}\right\}_{i \in \mathcal{A}}, V^{\Gamma}, \mathrm{p}^{\Gamma}\right\rangle$ is defined as follows:

1. $W^{\Gamma}=\left\{\Delta \mid \mathrm{p}^{\Delta}=\mathrm{p}^{\Gamma}\right.$ and $\Delta$ is an MCS. $\}$

2. For all $\Delta, \Delta^{\prime} \in W^{\Gamma}, \Delta R_{i}^{\Gamma} \Delta^{\prime}$ iff $\left\{\varphi \mid K_{i} \varphi \in \Delta\right\} \subseteq \Delta^{\prime}$

3. $V^{\Gamma}(p)=\left\{\Delta \in W^{\Gamma} \mid p \in \Delta\right\}$

(and $\mathrm{p}^{\Gamma}$ is defined according to Definition 8 ).

So, $\mathcal{P}^{\Gamma}$ is the canonical model constructed from the set of MCSs that agree with the protocol information in $\Gamma$. When there is no confusion, we omit the superscript $\Gamma$ in the defn of a canonical model. Now, given a maximally consistent set $\Gamma$ and a $\mathcal{L}_{D K A}$-formula $\varphi$, if $\langle\varphi\rangle \top \in \Gamma$, we define $\Gamma \varphi$ to be the set $\{\theta \mid\langle\varphi\rangle \theta \in \Gamma\}$. This can be lifted to sequences of formulas $\sigma$ as follows: $\left.\Gamma \sigma=\left(\ldots\left(\Gamma \sigma_{1}\right) \sigma_{2}\right) \ldots\right)$. We then have:

Lemma 1 For every maximally consistent set $\Gamma$ and sequence $\sigma$ of $\mathcal{L}_{D K A}$-formulas, $\Gamma \sigma$ (if defined) is a maximally consistent set.

Proof The proof is a simpleinduction on the length of $\sigma$. The key step uses standard modal reasoning to show from $\mathrm{R} 2$ and $\mathrm{R} 3$ that for any formula $\varphi$, (if defined) $\Delta \varphi$ is a maximally consistent set.

As usual, the key step is to prove a Truth Lemma. Much of the reasoning here is typical for modal completeness proofs. ${ }^{14}$ Nonetheless, our framework does raise some interesting issues which we focus on in this Section (leaving more standard arguments to the reader). The first stems from the fact that we restrict attention to maximally consistent sets that agree on the protocol information. Prima facie, this poses a problem for the "existence step" showing that if $K_{i} \varphi \notin \Gamma$ then there is an accessible state in the canonical model where $\varphi$ is false (i.e., $\varphi$ is not in the MCS). As usual, this state is constructed by showing that the set $\Delta^{\prime}=\left\{\psi \mid K_{i} \psi \in \Gamma\right\} \cup\{\neg \varphi\}$ is consistent and using Lindenbaum's Lemma to extend to a maximally consistent set $\Delta$. But how can we guarantee that $\Delta$ is in $W^{\Gamma}$ (i.e., $\left.\mathrm{p}^{\Gamma}=\mathrm{p}^{\Delta}\right)$ ? This is a direct consequence of Ptcl1 $\left(A_{i} \varphi \leftrightarrow K_{j} A_{i} \varphi\right)$ and $\operatorname{Ptcl} 2\left(\neg A_{i} \varphi \leftrightarrow K_{j} \neg A_{i} \varphi\right)$, as shown in the following lemma.

Lemma 2 Let $\Gamma$ be a maximally consistent set. Suppose $\Delta$ is a MCS such that $\left\{\psi \mid K_{i} \psi \in \Gamma\right\} \subseteq \Delta$. Then $\mathrm{p}^{\Gamma}=\mathrm{p}^{\Delta}$.

Proof Given Definition 8, it suffices to show that, for any sequence $\sigma$ of formulas in $\mathcal{L}_{D K A},\langle\sigma\rangle A_{i} \varphi \in \Gamma$ implies $\langle\tau\rangle A_{i} \varphi \in \Delta$, and $\langle\sigma\rangle \neg A_{i} \varphi \in \Gamma$ implies $\langle\tau\rangle \neg A_{i} \varphi \in \Gamma$, where $\tau$ is a sequence in $\mathcal{L}_{D K A}^{*}$ such that $\operatorname{len}(\tau)=\operatorname{len}(\sigma)$ and $\tau_{i}=\top$ for all $i(1 \leq i \leq \operatorname{len}(\sigma))$. The proof of the first implication appeals to Ptcl1, and the proof

\footnotetext{
14 We assume the reader is familiar with modal completeness proofs. For an excellent textbook presentation, see Section 4.2 of Blackburn et al. (2002).
} 
of the second appeals to Ptcl2. We only prove the first, since the proof of the second is similar.

By Uni, $\langle\sigma\rangle A_{i} \varphi \rightarrow[\tau] A_{i} \varphi$. Note here that, by standard DEL reasoning, $[\alpha] \beta \leftrightarrow$ $(\alpha \rightarrow\langle\alpha\rangle \beta)$ for any $\alpha, \beta \in \mathcal{L}_{D K A}$. Since $T$ is a tautology, it follows that $[\tau] A_{i} \varphi \leftrightarrow\langle\tau\rangle A_{i} \varphi$. Therefore, $\langle\sigma\rangle A_{i} \varphi \in \Gamma$ implies $\langle\tau\rangle A_{i} \varphi \in \Gamma$. Next, by standard modal reasoning, Ptcl1 implies $\langle\tau\rangle A_{i} \varphi \leftrightarrow\langle\tau\rangle K_{j} A_{i} \varphi$ and R4 implies that $\langle\tau\rangle K_{j} A_{i} \varphi \rightarrow K_{j}[\tau] A_{i} \varphi$. Therefore, $\langle\sigma\rangle A_{i} \varphi \in \Gamma$ implies $K_{j}[\tau] A_{i} \varphi \in \Gamma$. Given that $\top$ is a tautology again, this also yields $K_{j}\langle\tau\rangle A_{i} \varphi \in \Gamma$. Given the current assumption, we have $\langle\tau\rangle A_{i} \varphi \in \Delta$ as desired.

The second issue concerns the dynamic modalities ' $\langle\varphi\rangle$ ' and how to build an appropriate canonical model. This requires more thought since we must move from a single canonical model to the universe of all models generated from the initial canonical model by sequences of "making $\psi$ publicly available" events. In other words, we need to make use of models of the form $\left(\cdots\left(\left(\mathcal{P}^{\Gamma} \otimes \psi_{1}\right) \otimes \psi_{2}\right) \cdots \otimes \psi_{n}\right)$. To ease exposition, denote these models by $\mathcal{P}^{\Gamma} \sigma$ where $\sigma=\psi_{1} \cdots \psi_{n}$. The key idea is that since all necessary information is already available in the initial canonical model (as suggested by Lemma 1), we can simply use the update operation (Definition 6) to construct the additional models. To that end, we define the canonical models after a sequence of announcements:

Definition 10 (Canonical Model after $\sigma$ ) Given a (possibly empty) sequence $\sigma$ of $\mathcal{L}_{D K A}$-formulas and a $\Gamma$-generated canonical model $\mathcal{P}^{\Gamma}$, we define $\mathcal{P}^{\sigma}=$ $\left\langle W^{\sigma},\left\{R_{i}^{\sigma}\right\}_{i \in \mathcal{A}}, V^{\sigma}, \mathrm{p}^{\sigma}\right\rangle$ inductively as follows:

1. $\mathcal{P}^{\lambda}=\mathcal{P}^{\Gamma}(\lambda$ is the empty string $)$

2. $W^{\sigma_{(n)}}=\left\{\Delta \sigma_{n} \mid \mathcal{P}^{\sigma_{(n-1)}}, \Delta \models \sigma_{n}\right\} \cup\left\{\Delta \bar{\sigma}_{n} \mid \mathcal{P}^{\sigma_{(n-1)}}, \Delta \models \overline{\sigma_{n}}\right\}$

3. For all $\Delta \chi, \Delta^{\prime} \chi^{\prime} \in W^{\sigma_{(n)}}, \Delta \chi R_{i}^{\sigma_{(n)}} \Delta^{\prime} \chi^{\prime}$ iff

a) $\Delta R_{i}^{\sigma_{(n-1)}} \Delta^{\prime}$ and

b) either of the following array hold:

$$
\begin{aligned}
& -\chi=\chi^{\prime}, \text { or } \\
& -\bar{\chi}=\chi^{\prime} \text { and } \chi, \chi^{\prime} \notin \mathrm{p}^{\sigma_{(n-1)}}(i, 0)
\end{aligned}
$$

4. For all $p \in$ At and $\Delta \chi \in W^{\sigma_{(n)}}, \Delta \chi \in V^{\sigma_{(n)}}(p)$ iff $\Delta \in V^{\sigma_{(n-1)}}(p)$

5. $\mathrm{p}^{\sigma_{(n)}}(i, n):=\mathrm{p}^{\sigma_{(n-1)}}(i, n+1)$

A few comments are in order about the above definition. First of all, note that for any $\sigma$ and canonical model $\mathcal{P}, \mathcal{P}^{\sigma}$ is an epistemic model with a protocol according to Definition 5. Therefore, for any formula $\varphi$, we can use Definition 6 to interpret $\mathcal{P}^{\sigma}, \Delta \sigma \models \varphi$. Second, note also that $\mathcal{P}^{\sigma}=\mathcal{P}^{\bar{\sigma}}$ by the condition 2 above. Finally, recall that $\Delta \sigma$ is only defined provided $\langle\sigma\rangle \top \in \Delta$. Now, if $\langle\sigma\rangle \varphi \in \Delta$ then (by standard modal reasoning using $\mathrm{R} 1 \& \mathrm{R} 3)\langle\sigma\rangle \top \in \Delta$ and so $\Delta \sigma$ is defined. However, for an arbitrary sequence of DKA formulas $\sigma$, if $\mathcal{P}^{\sigma}, \Delta \sigma \models \chi$, we cannot conclude that $\Delta \sigma \chi$ is defined (this would require a Truth Lemma which we have not yet proved!). Thus in item 2 in the above Definition, there is an implicit assumption that each element of $W^{\sigma_{(n)}}$ is actually defined. It is important to keep this in mind in the remainder of this section.

Let us take stock of where we stand in the proof of completeness (Theorem 1). We need to show that any consistent set $\Gamma_{0}$ of DKA formulas has a model. Now, 
given a consistent set $\Gamma_{0}$ of DKA formulas, Lindenbaum's Lemma can be used to construct a maximally consistent set $\Gamma$ extending $\Gamma_{0}$. This MCS gives us a canonical model $\mathcal{P}^{\Gamma}=\left\langle W^{\Gamma},\left\{R_{i}^{\Gamma}\right\}_{i \in \mathcal{A}}, V^{\Gamma}, \mathrm{p}^{\Gamma}\right\rangle$ (Definition 9). Following the usual method for proving completeness, we must prove a Truth Lemma:

$$
\text { for all } \varphi \in \mathcal{L}_{D K A} \text {, for each } \Delta \in W^{\Gamma}, \varphi \in \Delta \text { iff } \mathcal{P}^{\Gamma}, \Delta \models \varphi \text {. }
$$

Much of the proof is a simple adaptation of the usual argument (some details are provided below). The difficulty comes when considering formulas of the form $\langle\sigma\rangle \psi$ where $\sigma$ is sequence of DKA formulas. We prove this by a (sub)induction on structure of $\psi$. The strategy is to use the canonical model after $\sigma$ (Definition 10). The following prop contains the key steps needed to complete the proof of the Truth Lemma:

Proposition 3 Let $\Gamma$ be a MCS and $\mathcal{P}^{\Gamma}=\left\langle W^{\Gamma},\left\{R_{i}\right\}_{i \in \mathcal{A}}, V^{\Gamma}, \mathrm{p}^{\Gamma}\right\rangle$ be a $\Gamma$-generated canonical model. For any sequence $\sigma \in \mathcal{L}_{D K A}^{*}$ :

1. For all $\Delta \in W^{\Gamma}$ (such that $\Delta \sigma$ is defined) and all $\varphi \in \mathcal{L}_{D K A}, \mathcal{P}^{\sigma}, \Delta \sigma \models \varphi$ iff $\mathcal{P}^{\Gamma} \otimes \sigma, \Delta \models \varphi$.

2. For all $\Delta \in W^{\Gamma}$ (such that $\Delta \sigma$ is defined) and all $\varphi \in \mathcal{L}_{D K A},\langle\sigma\rangle \varphi \in \Delta$ iff $\mathcal{P}^{\sigma}, \Delta \sigma \models \varphi$.

We prove this prop in a series of Lemmas. The first item follows from the following Lemma:

Lemma 3 Suppose $\mathcal{P}^{\Gamma}$ is a $\Gamma$-generated canonical model and $\sigma$ is a sequence of DKA formulas. For any $\Delta$ in $\mathcal{P}^{\sigma}$ and formula $\chi \in \mathcal{L}_{D K A}$ where $\Delta \chi$ is defined and $\mathcal{P}^{\sigma}, \Delta \models \chi$,

$$
\text { for all } \varphi \in \mathcal{L}_{D K A}, \quad \mathcal{P}^{\sigma \chi}, \Delta \chi \models \varphi \quad \text { iff } \quad \mathcal{P}^{\sigma} \otimes \chi, \Delta \models \varphi \text {. }
$$

Proof It suffices to show that, for all sequences $\sigma \in \mathcal{L}_{D K A}^{*}$, the two models, $\mathcal{P}^{\sigma}$ and $\left(\cdots\left(\mathcal{P} \otimes \sigma_{1}\right) \cdots \otimes \sigma_{\operatorname{len}(\sigma)}\right)$, are isomorphic, i.e. there is one to one map between the domains of the two models that preserves the accessibility relations $\left\{R_{i}\right\}_{i \in \mathcal{A}}$ and valuation $V$. (The protocols in the two models are clearly identical, given the condition 5 in Definition 10 and the defn of $\otimes$ operation in Definition 6.) We prove this by induction on len $(\sigma)$. The base case follows from Definition 10 (and the assumption that $\Delta \chi$ is defined). Suppose that the claim holds for an arbitrary $\sigma$. Then consider the models $\mathcal{P}^{\sigma} \otimes \chi$ and $\mathcal{P}^{\sigma \chi}$. For the inductive step, it suffices to show that there is an isomorphic map between $\mathcal{P}^{\sigma} \otimes \chi$ and $\mathcal{P}^{\sigma \chi}$. (By IH, $\mathcal{P}^{\sigma}$ and $\mathcal{P} \otimes \sigma$ are isomorphic.) Here, take a map $f$ from $\mathcal{P}^{\sigma} \otimes \chi$ to $\mathcal{P}^{\sigma \chi}$ so that $f(\Delta)=\Delta \chi$, if $\mathcal{P}^{\sigma} \otimes \chi, \Delta \models \chi ; f(\Delta)=\Delta \bar{\chi}$ if $\mathcal{P}^{\sigma} \otimes \chi, \Delta \models \bar{\chi}$. Clearly $\Delta \in V(p)$ iff $\Delta \chi \in V(p)$ by the condition 5 in Definition 10 and the defn of the $\otimes$ operation in Definition 6. For the accessibility relation, suppose $\Delta R_{i} \Delta^{\prime}$ in $\mathcal{P}^{\sigma} \otimes \chi$. This implies that $\Delta R_{i} \Delta^{\prime}$ in $\mathcal{P}^{\sigma}$. There are two array to consider.

Case 1 The truth values of $\chi$ at $\Delta$ and $\Delta^{\prime}$ are the same in $\mathcal{P}^{\sigma}$. In this case, we have $f(\Delta)=f\left(\Delta^{\prime}\right)$. This implies $\Delta \chi R_{i} \Delta \chi$ in $\mathcal{P}^{\sigma \chi}$ by the condition 2 and the first condition of $3 \mathrm{~b}$ in Definition 10. 
Case 2 The truth values of $\chi$ at $\Delta$ and $\Delta^{\prime}$ are different in $\mathcal{P}^{\sigma}$. In this case, by Definition 6 , we have to have $\chi \notin \mathrm{p}(i, 0)$. This implies $f(\Delta) R_{i} f\left(\Delta^{\prime}\right)$ in $\mathcal{P}^{\sigma \chi}$ by the condition 2 and the second condition of $3 \mathrm{~b}$ in Definition 10.

Therefore, $\Delta R_{i} \Delta^{\prime}$ in $\mathcal{P}^{\sigma} \otimes \chi$ implies $\Delta R_{i} \Delta^{\prime}$ in $\mathcal{P}^{\sigma \chi}$. The other direction of the implication is similar.

We need two more lemmas. These lemmas confirm that the canonical models after a sequence of updates (Definition 10) "keeps" enough structure to prove a truth lemma.

Lemma 4 For any $\sigma \in \mathcal{L}_{D K A}^{*}$, if $\Delta \sigma R_{i} \Delta^{\prime} \sigma^{\prime}$ in $\mathcal{P}^{\sigma}$ then

$$
\left\{\varphi \mid K_{i} \varphi \in \Delta \sigma\right\} \subseteq \Delta^{\prime} \sigma^{\prime} .
$$

Proof We prove the claim by induction on len $(\sigma)$. The base case is clear by Definition 9. Suppose that the claim holds of an arbitrary $\sigma$. Suppose $\Delta \sigma \chi R_{i} \Delta^{\prime} \sigma^{\prime} \chi^{\prime}$. For any $K_{i} \varphi \in \Delta \sigma \chi$, we have $\langle\chi\rangle K_{i} \varphi \in \Delta \sigma$ by definition. By R4, we have $A_{i} \chi \rightarrow$ $K_{i}[\chi] \varphi, \neg A_{i} \chi \rightarrow K_{i}([\chi] \varphi \wedge[\bar{\chi}] \varphi) \in \Delta \sigma$. We go by array.

Suppose $A_{i} \chi \in \Delta \sigma$. By Definition 10 (the condition $3 \mathrm{~b}$ ), we have $\chi=\chi^{\prime}$. This implies that $\chi \in \Delta^{\prime} \sigma^{\prime}$ (the presence of $\Delta^{\prime} \sigma^{\prime} \chi^{\prime}$ in the model implies $\chi^{\prime} \in \Delta^{\prime} \sigma^{\prime}$ by Definition 10). Also we have $K_{i}[\chi] \varphi \in \Delta \sigma$. By IH, we have $[\chi] \varphi \in \Delta^{\prime} \sigma^{\prime}$. Given $\chi,[\chi] \varphi \in \Delta^{\prime} \sigma^{\prime}$, we have $\langle\chi\rangle \varphi \in \Delta^{\prime} \sigma^{\prime}$, which implies $\varphi \in \Delta^{\prime} \sigma^{\prime} \chi^{\prime}$.

Suppose $\neg A_{i} \chi \in \Delta \sigma$. This implies that $K_{i}([\chi] \varphi \wedge[\bar{\chi}] \varphi) \in \Delta \sigma \chi$, which yields $K_{i}([\chi] \varphi), K_{i}[\bar{\chi}] \varphi \in \Delta \sigma \chi$ by standard modal reasoning. Here, if $\chi=\chi^{\prime}$, the argument goes similarly to the above argument. Thus suppose $\chi \neq \chi^{\prime}$, that is, $\bar{\chi}=\chi^{\prime}$. In this case, $\bar{\chi},[\bar{\chi}] \varphi \in \Delta^{\prime} \sigma^{\prime}$ (by the same reasoning as above). This will give us $\varphi \in \Delta^{\prime} \sigma^{\prime} \chi^{\prime}$.

Lemma 5 For any $\sigma \in \mathcal{L}_{D K A}^{*}$, if $\left\{\varphi \mid K_{i} \varphi \in \Delta \sigma\right\} \cup\{\psi\}$ is consistent, there is a maximally consistent set $\Delta^{\prime} \sigma^{\prime}$ such that $\left\{\varphi \mid K_{i} \varphi \in \Delta \sigma\right\} \cup\{\psi\} \subseteq \Delta^{\prime} \sigma^{\prime}$ and $\Delta \sigma R_{i} \Delta^{\prime} \sigma^{\prime}$ in $\mathcal{P}^{\sigma}$.

Proof The proof is by induction on len $(\sigma)$. The base case is clear by the standard completeness argument and Lemma 2 . The inductive step is given by applications of $\mathrm{R} 4$ that are similar to those given in the proof of Lemma 4.

We are now ready to prove Proposition 3 .

Proof (of Proposition 3) Let $\mathcal{P}^{\Gamma}=\left\langle W^{\Gamma},\left\{R_{i}\right\}_{i \in \mathcal{A}}, V^{\Gamma}, \mathrm{p}^{\Gamma}\right\rangle$ be a $\Gamma$-generated canonical model (where $\Gamma$ is a MCS). For part 1, we must show that for all sequences $\sigma \in \mathcal{L}_{D K A}^{*}$ and $\Delta \in W^{\Gamma}$ (such that $\Delta \sigma$ is defined) and all $\varphi \in \mathcal{L}_{D K A}, \mathcal{P}^{\sigma}, \Delta \sigma \models \varphi$ iff $\mathcal{P}^{\Gamma} \otimes \sigma, \Delta \models \varphi$. The proof is by induction on the length of the sequence $\sigma$ with the key inductive step following from Lemma 3.

For part 2, we must show that for all sequences $\sigma \in \mathcal{L}_{D K A}^{*}$ and $\Delta \in W^{\Gamma}$ (such that $\Delta \sigma$ is defined) and all $\varphi \in \mathcal{L}_{D K A},\langle\sigma\rangle \varphi \in \Delta$ iff $\mathcal{P}^{\sigma}, \Delta \sigma \models \varphi$. The proof is by induction on the complexity of $\varphi$. The base case is a direct consequence of Definitions 9 and 10 and axiom R1: $\langle\sigma\rangle p \in \Delta$ iff $\sigma_{1}, \sigma_{2}, \ldots, \sigma_{\operatorname{len}(\sigma)}, p \in \Delta$ iff $\Delta \in V(p), \Delta \sigma_{1} \in V^{\sigma_{1}}(p), \ldots, \Delta \sigma \in V^{\sigma}(p)$ iff $\mathcal{P}^{\sigma}, \Delta \sigma \models p$. The boolean array 
are as usual. The knowledge modality case follows by a standard argument using Lemmas 4 and 5 (cf. van Benthem et al. 2009; Hoshi 2009). Thus, we deal only with the array for $\langle\chi\rangle$ and $A_{i}$.

Suppose $\varphi$ is of the form $\langle\chi\rangle \psi$. Assume $\mathcal{P}^{\sigma}, \Delta \sigma \models\langle\chi\rangle \psi$. Then $\mathcal{P}^{\sigma}, \Delta \sigma \models \chi$ and $\mathcal{P}^{\sigma} \otimes \chi, \Delta \sigma \models \psi$. By Lemma 3, $\mathcal{P}^{\sigma \chi}, \Delta \sigma \chi \models \psi$. By the induction hypothesis, $\langle\sigma \chi\rangle \psi \in \Delta$. I.e., $\langle\sigma\rangle\langle\chi\rangle \psi \in \Delta$. For the left-to-right direction, assume $\langle\sigma\rangle\langle\chi\rangle \psi \in \Delta$. That is, $\langle\sigma \chi\rangle \psi \in \Delta$. Then, by the induction hypothesis, we have $\mathcal{P}^{\sigma \chi}, \Delta \sigma \chi \models \psi$. Also, since $\langle\sigma\rangle\langle\chi\rangle \psi \in \Delta$, using standard modal reasoning, we have $\langle\sigma\rangle\langle\chi\rangle \top \in \Delta$. Hence, by $\mathrm{R} 1,\langle\sigma\rangle \chi \in \Delta$. This implies that $\mathcal{P}^{\sigma}, \Delta \sigma \models \chi$. Applying Lemma 3, we have $\mathcal{P}^{\sigma} \otimes \chi, \Delta \sigma \models \psi$. Therefore, $\mathcal{P}^{\sigma}, \Delta \sigma \models\langle\chi\rangle \psi$, as desired.

Suppose $\varphi$ is of the form $A_{i} \psi$. First, assume $A_{i} \psi \in \Delta \sigma$. By defn, this means that $\left\langle\sigma_{n}\right\rangle \cdots\left\langle\sigma_{1}\right\rangle A_{i} \psi \in \Delta$. By Definition $4, \psi \in \mathrm{p}^{\Delta}(i, n)$. Recall that $\Gamma$ is the initial MCS used to construct the initial canonical model and that $\mathrm{p}^{\Gamma}=\mathrm{p}^{\Delta}$. By Definition 10 part 5, $\psi \in \mathrm{p}^{\sigma}(i, 0)$. Hence, $\mathcal{P}^{\sigma}, \Delta \sigma \models A_{i} \psi$. Now assume $\mathcal{P}^{\sigma}, \Delta \sigma \models A_{i} \psi$. Then $\psi \in \mathrm{p}^{\sigma}(i, 0)$. By Definition 10 part 5, this implies $\psi \in \mathrm{p}^{\Delta}(i, n)$ where $n$ is the length of $\sigma$. Also, by Definition 8, there are $\alpha_{1}, \ldots, \alpha_{n}$ such that $\left\langle\alpha_{1}\right\rangle \cdots\left\langle\alpha_{n}\right\rangle A_{i} \psi \in \Delta$. Then repeated applications of Uni and R2 gives us $\langle\sigma\rangle A_{i} \psi \in \Delta$.

Putting everything together, we now prove the main Truth Lemma:

Truth Lemma 6 For each formula $\varphi \in \mathcal{L}_{D K A}$, for each $\Delta \in W^{\Gamma}$, $\varphi \in \Delta$ iff $\mathcal{P}^{\Gamma}, \Delta \models \varphi$.

Proof The proof is by strong induction on the complexity of $\varphi, c(\varphi)$. If $c(\varphi)=0$ then $\varphi$ is an atomic prop and we have $p \in \Delta$ iff $\Delta \in V^{\Gamma}(p)$ iff $\mathcal{P}^{\Gamma} \models p$. Suppose the statement holds for all $\psi$ such that $c(\psi)<c(\varphi)$. There are four array:

1. $\varphi$ is of the form $\psi_{1} \wedge \psi_{2}$. The argument is completely standard.

2. $\varphi$ is of the form $\neg \psi$. The argument is completely standard.

3. $\varphi$ is of the form $K_{i} \psi$. The standard proof works given Lemma 2 .

4. $\varphi$ is of the form $A_{i} \varphi$. We have $A_{i} \varphi \in \Delta$ iff $\varphi \in \mathrm{p}^{\Gamma}(i, 0)$ iff $\mathcal{P}^{\Gamma}, \Delta \models A_{i} \varphi$.

5. $\varphi$ is of the form $\langle\sigma\rangle \psi$ where $\sigma$ is a sequence of DKA formulas. There are two array:

A. $\langle\sigma\rangle \top \in \Delta$. Then $\Delta \sigma$ is defined and the result follows from Proposition 3 .

B. $\langle\sigma\rangle \top \notin \Delta$. Then $\langle\sigma\rangle \psi \notin \Delta$. Hence the left-to-right direction is trivially true. Thus we need only show $\mathcal{P}^{\Gamma}, \Delta \not \models\langle\sigma\rangle \psi$. We show that for any sequence of formulas $\sigma$ with $\langle\sigma\rangle \top \notin \Delta$, there is some $i \leq \operatorname{len}(\sigma)$ such that $\mathcal{P}^{\Gamma}, \Delta \not \models$ $\left\langle\sigma_{(i)}\right\rangle \sigma_{i+1}$. If $\sigma=\chi$, we have $\langle\chi\rangle \top \notin \Delta$. This means $\chi \notin \Delta$. Since $c(\chi)<$ $c(\varphi)$ we have $\mathcal{P}^{\Gamma}, \Delta \not=\chi$. Suppose $\sigma=\sigma^{\prime} \chi$. If there is some $i \leq \operatorname{len}\left(\sigma^{\prime}\right)$ with $\mathcal{P}^{\Gamma}, \Delta \not \models\left\langle\sigma_{(i)}^{\prime}\right\rangle \sigma_{i+1}^{\prime}$ then we are done. Suppose no such $i$ exists. Then, since $\left\langle\sigma^{\prime} \chi\right\rangle \top \notin \Delta$, we have $\left\langle\sigma^{\prime}\right\rangle \chi \notin \Delta$. Since $c\left(\left\langle\sigma^{\prime}\right\rangle \chi\right)<c(\varphi)$, we have $\mathcal{P}^{\Gamma}, \Delta \forall\left\langle\sigma^{\prime}\right\rangle \chi$, as desired. Since $\langle\sigma\rangle \top \notin \Delta, \mathcal{P}^{\Gamma}, \Delta \not \models\left\langle\sigma_{(i)}\right\rangle \sigma_{i+1}$ but this means that for any $\psi, \mathcal{P}^{\Gamma} \otimes \sigma_{(i)}, \Delta \not \models\left\langle\sigma_{i+1} \cdots \sigma_{\text {len }(\sigma)}\right\rangle \psi$ and so $\mathcal{P}^{\Gamma}, \Delta \not \models$ $\langle\sigma\rangle \psi$.

As discussed above, the usual argument shows that every DKA-consistent set is satisfiable. This completes the proof of Theorem 1. 


\subsection{Describing protocols}

According to Definition 4, the notion of protocols is quite general. Indeed the only constraint on a protocol $\mathrm{p}$ is that $\varphi \in \mathrm{p}(i, n)$ iff $\bar{\varphi} \in \mathrm{p}(i, n)$ for each $i$ and $n$. Of course, other properties may be relevant. Many of these additional properties can be expressed using our language. For example, consider the following properties:

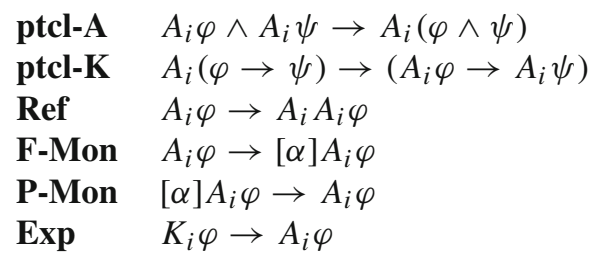

The first three properties are examples of structural properties of protocols. The first two (ptcl-A and ptcl-K) say that the protocol is closed under conjunction and consequence, respectively. The third property (Ref) says that if an agent has access to a formula $\varphi$, then the agent has access to that fact (i.e., $A_{i} \varphi$ ). Of course, these are only three examples of closure principles that may be of interest and many properties will be expressible using the $A_{i}$ modality. The next two properties (F-Mon and P-Mon) express monotonicity conditions of protocols toward future and past, respectively. That is, the axiom scheme F-Mon says that if a formula $\varphi$ is currently accessible, then it will be accessible after a future step. The converse (P-Mon) expresses a similar property looking into the past. Finally, the last axiom scheme (Exp) connects the agent's current information and access set. This can be understood as restricting to a type of explicit knowledge where agents must have access to what they (implicitly) know.

It is straightforward to show that the above sentences correspond to the mentioned properties. Given such correspondence results, adding these axiom schemes to the axiomatization DKA yields completeness proofs for classes of models based on protocols with the corresponding properties. Details are left to the reader.

\section{Discussion}

This paper has focused primarily on access sets interpreted as constraints on the agents' information gathering processes. That is, it is assumed there is some underlying protocol, or social convention, limiting the observations of the agents. We have discussed a logical framework for reasoning about such social interactive situations. Our main technical result is a sound and complete axiomatization, but we have also noted parallels between our frameworks and similar work found in the literature. Concrete translations from our logic to similar dynamic epistemic logics can be found in Sect. 3.2.

Perhaps more interesting are the broader themes discussed in Sect. 1. There we discussed a number of logical systems where access sets represent the set of formulas that are currently available to the agent. That is, access sets are the current outcome of some information gathering process rather than constraining the process. Despite similarities between the different formal frameworks, these two perspectives on the access sets are conceptually quite different. Nonetheless, merging the two perspectives may lead to new insights. We end by briefly discussing one such issue: dynamics. 
Much of the work on so-called inference logics (for example, see Ågotnes and Alechina 2007; van Benthem 2008; Velazquez-Quesada 2009, and references therein) is centered around the dynamic processes that change an agent's access set. The focus in much of the literature is on the modeling of agents that use logical inference rules to extend their access sets. However, the recent work of van Benthem (2008) and Velazquez-Quesada (2009) also use of DEL-style epistemic events to model change in the agents' access sets. But these dynamic operations also make sense in our context where the access sets are generated by some underlying protocol. While our protocols (Definition 4) do explicitly represent temporal shifts in the agents' access sets, we do not explicitly represent the dynamic events that cause these changes.

There are two levels where epistemic events can enter. The first is to think of the protocols as being generated by specific types of epistemic and/or "awareness" events. For example, use the "become informed" events of van Benthem (2008) to construct protocols where successive access sets are formed by execution of these events. This amounts to restricting attention to protocols satisfying specific structural properties (such as forwards monotonicity of access sets). Indeed, it would be interesting to analyze various structural properties on protocols (such as the ones discussed in Sect. 3.5) in terms of the type of events that generate protocols with those properties. But the protocols themselves may change as well. This is the second level where dynamic epistemic events can enter our analysis. Such dynamic operations are not as wellstudied (though see Renne et al. (2009); Icard et al. (2010) for some first steps in this direction) and we leave this for future work.

Acknowledgments The authors would like to thank the anonymous referees whose careful comments greatly improved the content and the readability of this text. The second author would also like to thank the participants at the workshop Formal Theories of Communication at the Lorentz Center in Leiden for their comments on an early version of this paper.

Open Access This article is distributed under the terms of the Creative Commons Attribution Noncommercial License which permits any noncommercial use, distribution, and reproduction in any medium, provided the original author(s) and source are credited.

\section{References}

Ågotnes, T., \& Alechina, N. (2007). The dynamics of syntactic knowledge. Journal of Logic and Computation, 17(1), 83-116.

Artemov, S. (2001). Explicit provability and constructive semantics. Bulletin for Symbolic Logic, 7(1), $1-36$.

Artemov, S., \& Nogina, E. (2005). Introducing justification into epistemic logic. Journal of Logic and Computation, 15(6), 1059-1073.

Balbiani, P., van Ditmarsch, H., \& Seban, P. (forthcoming). Reasoning about permitted announcements. Journal of Philosophical Logic.

Baltag, A., Moss, L., \& Solecki, S. (1998). The logic of common knowledge, public announcements and private suspicions. In I. Gilboa (ed.), Proceedings of the 7th conference on theoretical aspects of rationality and knowledge (TARK 98). (pp. 43-56).

Blackburn, P., Rijke, M., \& de Venema, Y. (2002). Modal logic. Cambridge: Cambridge University Press.

Eberle, R. (1974). A logic of believing, knowing and inferring. Synthese, 26, 356-382.

Fagin, R., \& Halpern, J. (1988). Belief, awareness and limited reasoning. Artificial Intelligence, 34, 39-76. 
Fagin, R., Halpern, J., Moses, Y., \& Vardi, M. (1995). Reasoning about knowledge. Boston: The MIT Press.

Fitting, M. (2005). The logic of proofs, semantically. Annals of Pure and Applied Logic, 132, 1-25.

Gerbrandy, J. (1999). Bisimulations on Planet Kripke. Ph.D. thesis, Institute for Logic, Language and Computation (DS-1999-01).

Halpern, J. (2001). Alternative semantics for unawareness. Games and Economic Behavior, 37, 321-339.

Halpern, J., \& Rego, L. (2005). Interactive unawareness revisited. In Proceedings of theoretical aspects of rationality and knowledge (TARK'05).

Halpern, J., \& Rego, L. (2009). Reasoning about knowledge of Unawareness Revisited. In A. Heifetz (ed.), Proceedings of theoretical aspects of rationality and knowledge (TARK'09).

Hill, B. (2010). Awareness dynamics. Journal of Philosophical Logic, 39(2), 113-137.

Hodkinson, I., \& Reynolds, M. (2006). Temporal logic. In P. Blackburn, J. van Benthem, \& F. Wolter (Eds.), Handbook of modal logic, vol. 3 of studies in logic (pp. 655-720). Amsterdam: Elsevier.

Hoshi, T. (2009). Epistemic dynamics and protocol information. Ph.D. thesis, Stanford University.

Hoshi, T., \& Yap, A. (2009). Dynamic epistemic logic with branching temporal structures. Synthese: Knowledge, Rationality, and Action, 169(2), 259-281.

Icard, T., Pacuit, E., \& Shoham, Y. (2010). Joint revision of beliefs and intentions. In F. Lin, U. Sattler, \& M. Truszczynski (Eds.), Principles of knowledge representation and reasoning: Proceedings of the twelfth international conference, KR 2010.

Kooi, B., \& Pacuit, E. (2010). Logics for rational interaction. In O. Roy, P. Girard, \& M. Marion (Eds.), Dynamic formal epistemology.

Pacuit, E. (forthcoming). Logics of informational attitudes and informative actions. Journal of the Indian Council of Philosophy. Available at http://ai.stanford.edu/ epacuit/papers/india-logknowbel.pdf

Parikh, R., \& Ramanujam, R. (2003). A knowledge based semantics of messages. Journal of Logic, Language and Information, 12, 453-467.

Plaza, J. (1989). Logics of public communications. In M. L. Emrich, M. S. Pfeifer, M. Hadzikadic, \& Z. Ras (Eds.), Proceedings, 4th international symposium on methodologies for intelligent systems. (pp. 201-216) (republished as Plaza 2007).

Plaza, J. (2007). Logics of public communications. Synthese: Knowledge, Rationality, and Action, $158(2), 165-179$.

Renne, B. (2008). Dynamic epistemic logic with justification. Ph.D. thesis, The City University of New York.

Renne, B., Sack, J., \& Yap, A. (2009). Dynamic epistemic temporal logic. In X. He, J. Horty, \& E. Pacuit (Eds.), Logic, rationality and interaction: Proceedings of LORI 2009, vol LNAI 5834. (pp. 263-277).

van Benthem, J. (1996). Exploring logical dynamics. CSLI Publications.

van Benthem, J. (2008). Merging observation and access in dynamic logic. Studies in Logic, 1, 1-17.

van Benthem, J. (2010). Logical dynamics of information flow. Cambridge: Cambridge University Press.

van Benthem, J., Gerbrandy, J., Hoshi, T., \& Pacuit, E. (2009). Merging frameworks for interaction. Journal of Philosophical Logic, 38(5), 491-526.

van Ditmarsch, H., \& French, T. (2010). Becoming aware. Manuscript.

van Ditmarsch, H., Hoek, W., \& van der Kooi, B. (2007). Dynamic epistemic logic. Synthese library. New York: Springer.

Velazquez-Quesada, F. R. (2009). Inference and update. Synthese (Knowledge, Rationality \& Action), 169(2), 283-300. 